\title{
Joint Design of Network Coding and Channel Decoding for Wireless Networks
}

\author{
Shengli Zhang ${ }^{\dagger}$, Yu Zhu ${ }^{*}$, Soung-Chang Liew ${ }^{\dagger}$, and Khaled Ben Letaief* \\ ${ }^{\dagger}$ Dept. of Information Engineering \\ The Chinese University of Hong Kong \\ New Territories, HK SAR, China \\ \{slzhang5, soung\}@ie.cuhk.edu.hk \\ *Center for Wireless Information Technology \\ Department of Electronic and Computer Engineering \\ The Hong Kong University of Science \& Technology \\ Clear Water Bay, Kowloon, Hong Kong \\ \{eezhuyu, eekhaled \}@ece.ust.hk
}

\begin{abstract}
Network coding has been receiving much attention recently for its ability to improve network throughput and enhance network robustness. In this paper, we investigate the design of network coding in wireless networks and propose a combined low complexity network coding and channel decoding scheme. We analyze the capacity of the proposed scheme for both the binary symmetric channel (BSC) and AWGN channel and show that it can achieve almost the same channel capacity as traditional network coding with a small degradation in the system bit error rate (BER) performance while achieving almost $50 \%$ complexity reduction. It is also shown that the proposed network coding design can be applied in wireless cooperative networks.
\end{abstract}

Keywords: Network Coding, Channel Decoding, Wireless Networks, Log-Likelihood Ratio.

\section{INTRODUCTION}

Network coding has recently received much attention from the research community because of its potential for improving network throughput and robustness. In a wireline network with network coding, the intermediate relay nodes between the source and the destination nodes may process and mix the data packets from different inputs to produce output packets before forwarding them. It has been shown that with network coding, the wireline packet network can achieve the max-flow min-cut throughput for multicast transmissions [1]. It has also been shown in [2] that this capacity can be achieved by applying linear encoding at the intermediate nodes.

Motivated by these attractive results, recent research efforts have been extended to network coding in wireless networks. In such networks, the packet sent by a node can be detected by several other nodes simultaneously. This broadcast property, referred to as the wireless multicast advantage [4], makes the use of network coding in wireless networks even more natural [5]. In [3], it was shown that with network coding, low-complexity and energy-efficient routing algorithms can be designed. [6] showed that network throughput can be significantly improved by using opportunistic network coding that takes into account the changing wireless environments. Likewise, [7] showed that network coding is much more robust against packet loss than conventional schemes.

The design of network coding has also been considered in the physical layer of wireless networks and was combined with the techniques that deal with the detrimental effects of wireless channel fading. For example, the joint design of network coding and channel coding has been considered in $[8,9]$ to obtain additional diversity gain. Another example is the physical layer network coding where direct network coding by the mixing of EM (electromagnetic) signals (as opposed to "bits" in the digital domain) has been shown in [10] to obtain further increases in network throughput. In contrast to previous work, this paper considers an alternative joint network coding and channel decoding design at the intermediate nodes. In traditional network coding, packets received from different nodes are first decoded separately and then combined by network coding. In the proposed scheme, however, and by taking the linear property of the channel coding and network coding into account, network coding is performed prior to the channel decoding by combining the hard or soft decisions of each received packets. It is shown that the proposed scheme can be seen as an alternative to the traditional network coding scheme to improve the wireless network throughput. We analyze the capacity of the proposed scheme for both the binary symmetric channel (BSC) and AWGN channel and show that the proposed scheme can achieve almost the same channel capacity as the traditional one at moderate and large SNR values. It is also shown by simulation that the performance degradation of the proposed scheme is around $0.5 \sim 1 \mathrm{~dB}$ at a bit error rate (BER) of $10^{-5}$ in AWGN channels. However, compared to the traditional network coding scheme, only one channel decoding process is needed and the computational complexity at the intermediate node is reduced by almost $50 \%$.

The rest of this paper is organized as follows. In Section II, we present the system model along with a brief description of the traditional network coding scheme in wireless networks. Section III describes the joint network coding and channel decoding design with soft and hard detection. In section IV, the channel capacity of the proposed scheme is analyzed and compared with that of the traditional scheme. Section $\mathrm{V}$ presents numerical results. We discuss the application of the proposed network coding design for wireless cooperative networks in Section VI and conclude the paper in Section VII.

\section{SYSTEM MODEL}

Throughout this paper we focus on a typical network coding unit as described in many prior investigations (e.g., [1-3]), as 
shown in Fig. 1. Nodes $n_{1}$ and $n_{2}$ are two independent source nodes and node $n_{3}$ is the intermediate node. At $n_{3}$, two packets, $X_{1}$ and $X_{2}$ from $n_{1}$ and $n_{2}$, respectively, are linearly combined by network coding and forwarded to other nodes. Typically, the network coding combination takes the form of an exclusive OR operation, i.e., XOR.

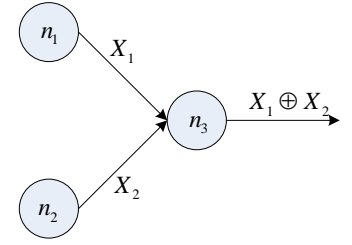

Fig. 1. Typical network coding unit.

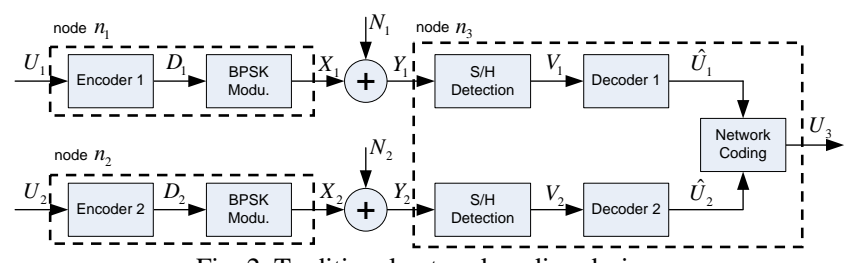

Fig. 2. Traditional network coding design.

Fig. 2 depicts the signal processing procedures in traditional networking coding. Let $U_{i}=\left[u_{i, 0}, u_{i, 1}, \cdots u_{i, K-1}\right]$ denote the data bits in the information packet of node $n_{i}$. It is assumed that the packets from $n_{1}$ and $n_{2}$ have the same packet length $K$ and the binary information bits $u_{i, k} \in\{0,1\}$ for $k=0, \ldots, K-1$ are independent and uniformly distributed. At each node, it is assumed that the information packet is coded using the same channel coding scheme, with a reversible mapping function $\Gamma$. The relationship between the information packet and the codeword $D_{i}=\left[d_{i, 0}, d_{i, 1}, \ldots, d_{i, N-1}\right]$, where $d_{i, n} \in\{0,1\}$ for $i=1,2$, can be represented by

$$
\Gamma\left(U_{i}\right)=D_{i} \quad \Gamma^{-1}\left(D_{i}\right)=U_{i}
$$

where the $\Gamma^{-1}$ denotes the decoding processing. It is easy to see that the coding rate is $K / N$.

We assume that BPSK modulation is used and that the mapping from the coded bit $d_{i, n}$ to the BPSK symbol $x_{i, n}$ is given by

$$
x_{i, n}=1-2 d_{i, n} .
$$

For simplicity, it is assumed that the modulated packet, $X_{i}$, is transmitted to node $n_{3}$ through AWGN channels. That is,

$$
y_{i, n}=x_{i, n}+n_{i, n} \quad \text { for } i=1,2 \text { and } n=1, \ldots, N
$$

where $n_{i, n}$ is the white Gaussian noise with variance $\sigma^{2}$. It should be noted here that our proposed scheme can be easily generalized to systems with other modulation schemes and wireless fading channels.

At the intermediate node $n_{3}$, let $v_{i, n}$ for $i=1,2$ and $n=1, \ldots, N$ denote the hard/soft decision on the received signal $y_{i, n}$. If the receiver performs hard detection, we have

$$
v_{i, n}=\operatorname{sign}\left(y_{i, n}\right) \text {. }
$$

If soft detection is applied, then soft decision, which is usually represented by the Log-Likelihood Ratio (LLR), will be generated and sent to the channel decoder, where some algorithms, such as BCJR [11] and LogMAP [12], may be used. With reference to [12], $v_{i, n}$ is given by

$$
\begin{aligned}
& v_{i, n}=\ln \left(\frac{P\left(x_{i, n}=1 \mid y_{i, n}\right)}{P\left(x_{i, n}=-1 \mid y_{i, n}\right)}\right)=\ln \left(\frac{P\left(y_{i, n} \mid x_{i, n}=1\right)}{P\left(y_{i, n} \mid x_{i, n}=-1\right)}\right) \\
& =2 y_{i, n} / \sigma^{2} \quad \text { for } i=1,2 \text { and } n=0, \ldots, N-1 .
\end{aligned}
$$

Finally, the hard-input hard-output (HIHO) or soft-input hard-output (SIHO) algorithms can be used to decode the signal. Let $\hat{U}_{1}$ and $\hat{U}_{2}$ denote the estimation of packet $U_{1}$ and $U_{2}$. The network coded packet, $U_{3}$, is obtained by

$$
U_{3}=\hat{U}_{1} \oplus \hat{U}_{2}
$$

where “ $\oplus$ ” denotes the XOR operation.

\section{JOINT NETWORK CODING AND CHANNEL DECODING DESIGN}

\section{A. Basic idea}

In contrast to the traditional network coding scheme, where network coding is performed after the channel decoding processing, in the proposed scheme, as shown Fig. 3, network coding is performed prior to the channel decoding processing by directly combining the soft/hard decisions.

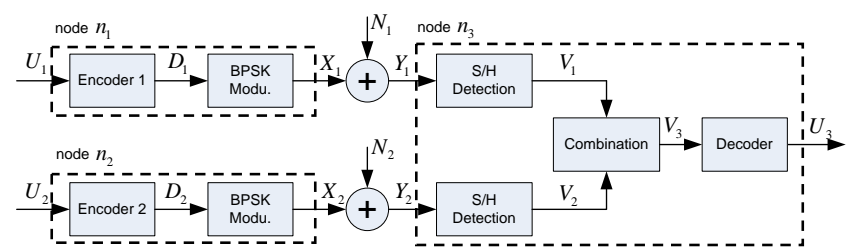

Fig. 3. Joint network coding and channel decoding design.

The basic idea stems from the linear property of the channel code. That is, the linear combination of the two codewords, which are generated from exactly the same coding scheme with the same length, is actually another codeword. This linearity can be formulated as

$$
\Gamma\left(U_{1} \oplus U_{2}\right)=\Gamma\left(U_{1}\right) \oplus \Gamma\left(U_{2}\right) .
$$

Almost all of the practical wireless channel codes, such as convolutional codes, Turbo codes, and LDPC codes, are linear codes. By applying this property of the channel codes, and the fact that network coding is also a linear mapping, it is easily seen that the order of these two processes can be exchanged. This motivates the proposed scheme, as shown in Fig. 3. By carefully combining the hard/soft decisions, $V_{1}$ and $V_{2}$, the output signal $V_{3}$ can be used to decode the target information packet $\left(U_{1} \oplus U_{2}\right)$. In the special case where there is no transmission error, our design can be expressed simply by

$$
\begin{aligned}
& U_{3}=\Gamma^{-1}\left(V_{3}\right)=\Gamma^{-1}\left(V_{1} \oplus V_{2}\right)=\Gamma^{-1}\left(D_{1} \oplus D_{2}\right) \\
& =\Gamma^{-1}\left(\Gamma\left(U_{1}\right) \oplus \Gamma\left(U_{2}\right)\right)=\Gamma^{-1} \Gamma\left(U_{1} \oplus U_{2}\right)=U_{1} \oplus U_{2}
\end{aligned} .
$$

By comparing the schemes in Fig. 2 and Fig. 3, we see that the proposed scheme can perform network coding with only one channel decoding process while the traditional scheme requires two channel decoding processes. Since most of the power in baseband signal processing is consumed by the 
channel decoder, the proposed scheme can greatly increase the power efficiency of the intermediate nodes in wireless networks.

\section{B. Design with hard detection}

If hard detection is used at the intermediate node as in (4), the detected packet $V_{i}$ with $v_{i, n} \in\{-1,+1\}$ can be represented by

$$
V_{i}=X_{i} * E_{i} \quad \text { for } i=1,2
$$

where $*$ denotes the multiplication operation at each element, and $E_{i}=\left[e_{i, 0}, e_{i, 1}, \ldots, e_{i, N-1}\right]$ with $e_{i, n} \in\{-1,+1\}$ represents the binary error pattern, where $e_{i, n}=1$ corresponds to the correct reception of the coded bit $x_{i, n}$ and $e_{i, n}=-1$ corresponds to the error reception. It is easy to see that the combination in this case is to multiply the corresponding element in the detected packets. That is,

$$
\begin{aligned}
& V_{3}=V_{1} * V_{2}=\left(X_{1} * E_{1}\right) *\left(X_{2} * E_{2}\right) \\
& =\left(X_{1} * X_{2}\right) *\left(E_{1} * E_{2}\right)
\end{aligned} .
$$

It should be noted that in the case of BPSK modulation, $X_{1} * X_{2}$ corresponds to $D_{1} \oplus D_{2}$. In this case $V_{3}$ can be regarded as the output of a virtual BSC channel where the equivalent input is $X_{1} * X_{2}$ and the error in this virtual channel is $E_{1} * E_{2}$.

\section{Design with soft detection}

When soft detection is used, network coding is performed by combining the soft decisions (5) of the two transmitted packets and maintaining as much information as possible. Specifically, such combination is expressed as

$$
\begin{aligned}
& v_{3, n}=\ln \left(\frac{P\left(d_{1, n} \oplus d_{2, n}=0 \mid y_{1, n}, y_{2, n}\right)}{P\left(d_{1, n} \oplus d_{2, n}=1 \mid y_{1, n}, y_{2, n}\right)}\right) \\
& =\ln \left(\frac{P\left(x_{1, n} * x_{2, n}=1 \mid y_{1, n}, y_{2, n}\right)}{P\left(x_{1, n} * x_{2, n}=-1 \mid y_{1, n}, y_{2, n}\right)}\right) . \\
& =\ln \left(\frac{1+\exp \left(v_{1, n}+v_{2, n}\right)}{\exp \left(v_{1, n}\right)+\exp \left(v_{2, n}\right)}\right)
\end{aligned}
$$

It can be easily seen that $v_{3, n}$ actually denotes the LLR of the bit $d_{1, n} \oplus d_{2, n}$. It should be noted here that in contrast to the traditional network coding scheme, where the combination is the addition operation in the finite field $G F(2)$, the proposed combination calculates the soft information (i.e., LLR) of the network coded bit. Thus, we refer to it as soft network coding.

A clearer explanation of the soft network coding in (11) can be obtained by using the following approximation

$$
\begin{aligned}
& v_{3, n}=\ln \left(\frac{1+\exp \left(v_{1, n}+v_{2, n}\right)}{\exp \left(v_{1, n}\right)+\exp \left(v_{2, n}\right)}\right) \\
& \approx \operatorname{sign}\left(v_{1, n}\right) \operatorname{sign}\left(v_{2, n}\right) \min \left(\left|v_{1, n}\right|,\left|v_{2, n}\right|\right) \\
& =\left(x_{1, n} * x_{2, n}\right) *\left(e_{1, n} * e_{2, n}\right) \min \left(\left|v_{1, n}\right|,\left|v_{2, n}\right|\right)
\end{aligned}
$$

where $e_{i, n}$ denotes the element in the error pattern $E_{i}$ in the hard detection case. It is clearly shown in (12) that the soft network coding output can be approximated by the combination result in the hard detection case in (10) weighted by $\min \left(\left|v_{1, n}\right|,\left|v_{2, n}\right|\right)$. A closer observation on (10) and (12) indicates that the noise effect is enhanced after the combination and more decision errors are generated, compared to the traditional scheme. In Section V, some simulation results will be provided to show that around $0.5 \sim 1.0 \mathrm{~dB}$ more transmit power is required to achieve the same error probability.

We conclude this section by noting that the proposed soft network coding idea can be generalized to many prospective applications. For example, the soft network coded packet can be forwarded directly to the destination node for the final joint decoding in a wireless relay network. This design is similar to the amplify-forward method in the relay transmission and in some situation can achieve better performance than the traditional design, while greatly reducing the complexity of the relay node.

\section{CAPACITY ANALYSIS}

The previous section has shown that although the proposed scheme reduces the decoding complexity, the enhanced noise effect may decrease the system performance. In this section, we analyze the channel capacity of the proposed scheme and compare it to that of the traditional approach. The channel discussed here is defined as the equivalent channel whose input is $X_{1} * X_{2}$. It is assumed that the two transmission channels between the source nodes and the intermediate node are independent and with the same random properties. In this case, the capacity of the equivalent channel is equal to that of the transmission channel for the traditional design.

\section{A. Capacity in the BSC channel}

When hard detection is used, the BSC channel model can be applied here to describe the transmission, where the crossover error probability $p$ of the BSC channel is equal to the BER of the real transmission. For example, in the system with BPSK modulation and AWGN channel, the cross-over error probability is given by [13]

$$
p\left(e_{1, n}=-1\right)=p\left(e_{2, n}=-1\right)=p=Q\left(\sqrt{2 E_{b} / N_{0}}\right) .
$$

The capacity of the equivalent channel in the traditional scheme is equal to that of the transmission channel between nodes $n_{1} / n_{2}$ and $n_{3}$, which is also equal to the capacity of the BSC channel. Therefore, we can obtain the channel capacity according to [14]

$$
\mathrm{C}_{\mathrm{T}, \mathrm{BSC}}=1-\mathrm{H}(p)
$$

where $\mathrm{H}(p)=-p \log _{2} p-(1-p) \log _{2}(1-p)$ is the entropy of binary distribution with probability $p$.

It can be drawn from (10) that the capacity of the proposed design can be obtained by considering the capacity of the equivalent virtual BSC channel with the error pattern $E_{1} * E_{2}$. The crossover error probability of this equivalent BSC channel is given by 


$$
\begin{aligned}
& p_{1}=P\left(e_{1, n} * e_{2, n}=-1\right) \\
& =P\left(e_{1, n}=1, e_{2, n}=-1\right)+P\left(e_{1, n}=-1, e_{2, n}=1\right) . \\
& =2 p(1-p)
\end{aligned}
$$

Thus, we have the capacity of the proposed design as

$$
\mathrm{C}_{\mathrm{N}, \mathrm{BSC}}=1-\mathrm{H}\left(p_{1}\right)=1-\mathrm{H}\left(2 p-2 p^{2}\right) \text {. }
$$

Since in practical systems $p<1 / 2$, it is easy to see that $p_{1}>p$. By substituting this relation into (16), we can see that the capacity of the proposed design is less than that of the traditional design.

\section{B. Capacity in AWGN channel}

We now assume that the packets are transmitted in AWGN channels and that soft detection is used at the intermediate node. As we mentioned above, the capacity of the equivalent channel in the traditional design is equal to that of the real transmission channel, which is given as follows according the definition of mutual information [14, 15]

$$
\mathrm{C}_{\mathrm{T}, \mathrm{AWGN}}=\sum_{x_{i, n}= \pm 1} \int_{y_{i, n}} p\left(y_{i, n}, x_{i, n}\right) \log _{2}\left(\frac{p\left(y_{i, n} \mid x_{i, n}\right)}{\sum_{\tilde{x}= \pm 1} p\left(y_{i, n} \mid \tilde{x}\right)}\right) d y_{i, n}
$$

where $p\left(y_{i, n}, x_{i, n}\right)$ and $p\left(y_{i, n} \mid x_{i, n}\right)$ denote the joint and the conditional probability density function (PDF) between $y_{i, n}$ and $x_{i, n}$, respectively. By following the same derivation procedure of [15], we have (17) as

$$
\begin{aligned}
& \mathrm{C}_{\mathrm{T}, \mathrm{AWGN}}=E\left\{\log _{2}\left(\frac{2}{1+\exp \left(-2 y_{i, n} / \sigma^{2}\right)}\right) \mid x_{i, n}=1\right\} \\
& =E\left\{\log _{2}\left(\frac{2}{1+\exp \left(-v_{i, n}\right)}\right) \mid x_{i, n}=1\right\}
\end{aligned}
$$

where the expectation operation is taken with respect to $y_{i, n}$.

The capacity of the equivalent channel in the proposed scheme can be obtained by calculating the mutual information between the input signal $X_{1} * X_{2}$ and the output signal $V_{3}$. That is,

$$
\begin{aligned}
& \mathrm{C}_{\mathrm{N}, \mathrm{AWGN}}= \\
& \sum_{\substack{x_{1, n} * x_{2, n} \\
= \pm 1}} \int_{v_{3, n}} p\left(v_{3, n}, x_{1, n} * x_{2, n}\right) \log _{2}\left(\frac{p\left(v_{3, n} \mid x_{1, n} * x_{2, n}\right)}{\sum_{\tilde{x}_{1, n} * \tilde{x}_{2, n}= \pm 1} p\left(v_{3, n} \mid \tilde{x}_{1, n} * \tilde{x}_{2, n}\right)}\right) d v_{3, n}
\end{aligned}
$$

where $v_{3, n}$ is given in (12). It turns out that it is very difficult to obtain the PDF of $v_{3, n}$. However, through simulations, we find that $v_{3, n}$ can be approximated as Gaussian distribution with a larger variance. With this approximation, we have

$$
C_{\mathrm{N}, \mathrm{AWGN}}=E\left\{\log _{2}\left(\frac{2}{1+\exp \left(-v_{3, n}\right)}\right) \mid x_{1, n} * x_{2, n}=1\right\} \text {. }
$$

The numerical results of the comparison of the capacity between the traditional and the proposed designs will be provided in Section V.

\section{NUMERICAL RESULTS}

This section presents sample numerical results to demonstrate the potential of the proposed joint network coding and channel decoding design.

\section{A. Capacity}

The numerical results of the capacity analysis introduced in Section IV are provided in Fig. 4. To evaluate the capacity in (18) and (20), we use the method in Appendix-I of [15]. It can be seen from this figure that the capacity of the traditional or the proposed scheme in the BSC channel is smaller than its capacity in AWGN channel. This is because hard detection, which leads to the information loss, is used in the BSC channel case, while soft detection is assumed in the case of AWGN channel.

It is also shown in Fig. 4 that the proposed design has lower capacity than the traditional one. This is because the combination prior to the channel coding enhances the noise effect and increases the error probability. For example, in the BSC channel, it is easy to see from (10) that, the number of detection errors is almost doubled after the combination. This is also true in the AWGN channel case. As seen in (12), the magnitude of the output LLR of the combination is approximately equal to the minimum one of the two input LLRs, which corresponds to a larger noise variance. However, as seen from Fig. 4, as SNR increases, the performance loss will decrease. In the moderate and high region of SNR values, the proposed design can achieve almost the same capacity as the traditional one.

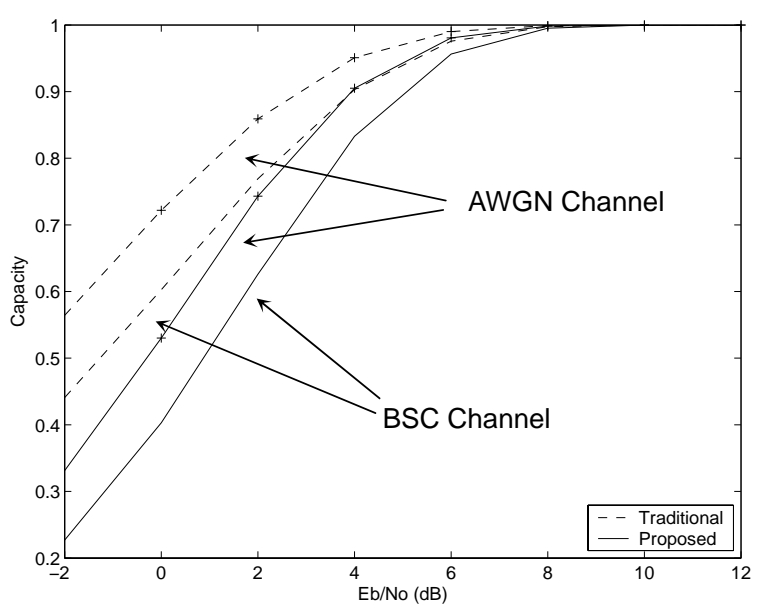

Fig. 4. Capacity comparison of the proposed joint network coding and channel decoding design and the traditional network coding design.

\section{B. Bit Error Probability}

We now compare the schemes from the transmission error probability point of view. It is assumed that all links are AWGN channels with the same noise variance. The standard convolutional code with rate $1 / 2$, constraint length 5 , and octal generator polynomials $(23,35)$ is applied. It is also assumed that the number of information bits in a packet is 200. Fig. 5 presents the performance results of the traditional and the 
proposed designs in two cases: hard decisions input and soft decisions input. For the proposed design, in the hard decisions input case, the hard decisions to the received signals from two source nodes are combined according to (10) before the Viterbi decoding. In the soft decisions input case, the soft decisions to the received signals from two source nodes are soft network coded according to (12) and then passed to the soft Viterbi decoder. It can be seen from Fig. 5 that the performance of the proposed design approaches to that of the traditional one as SNR increases. For example, there is only $0.6 \mathrm{~dB}$ and $1.1 \mathrm{~dB}$ difference between the two schemes at a BER of $10^{-5}$ for the hard decisions input case and the soft decisions input case, respectively.

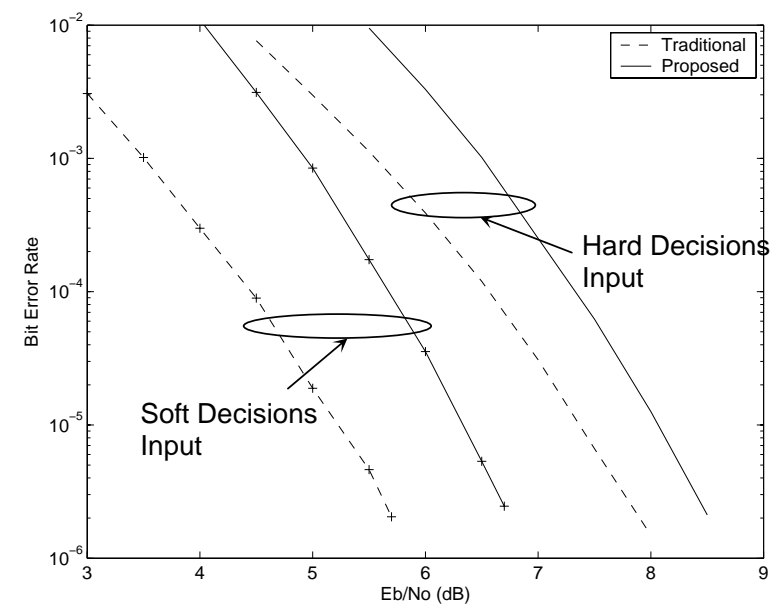

Fig. 5. Performance comparison of the proposed joint network coding and channel decoding design and the traditional network coding design in both hard and soft decisions input cases.

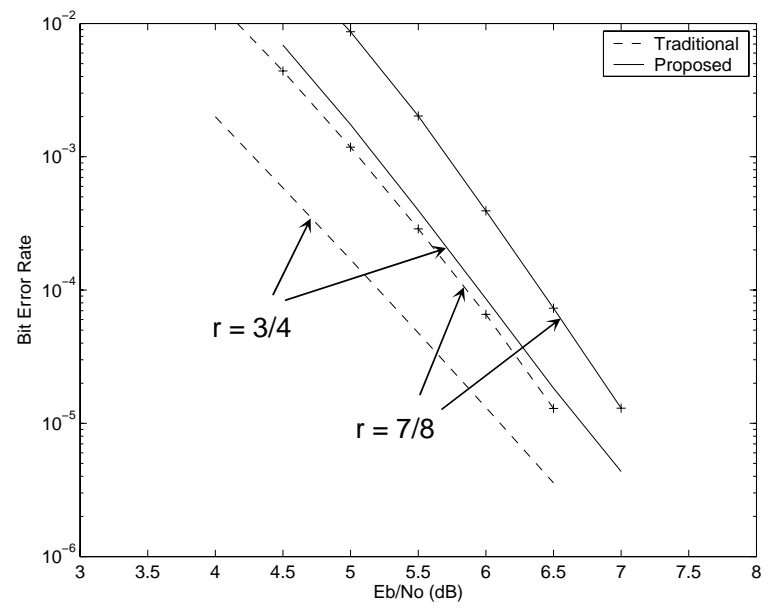

Fig. 6. Performance comparison of the proposed joint network coding and channel decoding design and the traditional network coding design for different coding rates.

In Fig. 6, we investigate the performance of the proposed design with soft decisions input for different coding rates. Two coding rates, $3 / 4$ and $7 / 8$, are considered. The number of information bits per packet is again 200. These two coding rates are realized by optimally puncturing [16] the above standard convolutional code of rate 1/2. It can be seen in Fig. 6 that the proposed scheme performs closer to the traditional scheme as coding rate increases. For example, at a BER of $10^{-5}$, compared to $1.1 \mathrm{~dB}$ SNR gap in the rate $1 / 2$ case in Fig. 5 , there is only $0.5 \mathrm{~dB}$ and $0.6 \mathrm{~dB}$ difference between the two schemes, for rate $3 / 4$ and rate $7 / 8$, respectively. This is because for high coding rate cases, in order to achieve the same BER, more bit energy is required. This makes the soft decisions more reliable and reduces the performance loss in the soft network coding.

\section{APPLICATION}

We have shown in the previous sections that the proposed soft network coding can be easily combined with channel decoding to save $50 \%$ computational complexity with only a small degradation in BER performance. In this section we show that the proposed soft network coding can be further applied to wireless cooperative networks.

We consider a two-way cooperative network as shown in Fig. 7, which was also considered in [8]. In this network, node $n_{1}$ and $n_{2}$ exchange their packets with the help of node $n_{3}$. In the first time slot $n_{1}$ broadcasts its packet $x_{1}$, and in the second time slot $n_{2}$ broadcasts its packet $x_{2}$. Thanks to the network coding technique, node $n_{3}$ can perform the relay transmission for both node $n_{1}$ and $n_{2}$ in only one time slot by broadcasting the network coded packet $x_{1} \oplus x_{2}$.

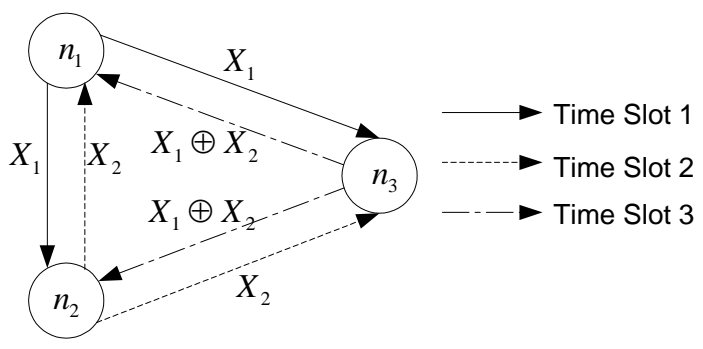

Fig. 7. Network coding design in a two-way wireless cooperative network.

Let $y_{i}^{j}$ and $n_{i}^{j}$ denote the received signal and the noise signal at node $n_{j}$, respectively, when $x_{i}$ is transmitted. Then, we have

$$
\begin{aligned}
& y_{1}^{3}=x_{1}+n_{1}^{3} \\
& y_{2}^{3}=x_{2}+n_{2}^{3} .
\end{aligned}
$$

In contrast to the previous work in [8], in our design, the relay node $n_{3}$ performs the soft network coding and broadcasts the soft network coded signal to both node $n_{1}$ and node $n_{2}$. According to (12), the soft network coded signal is given by

$$
x_{3} \approx \operatorname{sign}\left(y_{1}^{3} \cdot y_{2}^{3}\right) \min \left(\left|y_{1}^{3}\right|,\left|y_{2}^{3}\right|\right) .
$$

As shown in Section IV, $x_{3}$ can be regarded as the transmission of $x_{1} \oplus x_{2}$ in a virtual Gaussian channel. That is,

$$
x_{3}=x_{1} \cdot x_{2}+n_{v}^{3}
$$

where $n_{v}^{3}$ denotes the equivalent noise component in the virtual channel. The received signal of $x_{3}$ at node $n_{1}$ can be given by

$$
y_{3}^{1}=x_{3}+n_{3}^{1} \text {. }
$$


$n_{1}$ can retrieve the information in $x_{2}$ from the received signal $y_{3}^{1}$ by combining $y_{3}^{1}$ and it own packet $x_{1}$ with soft network coding (note that the magnitude of the LLR value of $x_{1}$ at node $n_{1}$ is infinite), which is given by

$$
\begin{aligned}
y_{d}^{1} & \approx \operatorname{sign}\left(x_{1} \cdot y_{3}^{1}\right) \min \left(\left|x_{1}\right|,\left|y_{3}^{1}\right|\right) \\
& =\operatorname{sign}\left(x_{1} \cdot y_{3}^{1}\right)\left|y_{3}^{1}\right|
\end{aligned} .
$$

It should be noted here that in the second time slot node $n_{1}$ has got another copy of the signal $x_{2}$

$$
y_{2}^{1}=x_{2}+n_{2}^{1} \text {. }
$$

After combining the two copies of the packet $x_{2}$, i.e., $y_{d}^{1}$ and $y_{2}^{1}$, with the maximum ratio combination (MRC) criterion, node $n_{1}$ will obtain a more reliable received signal of $x_{2}$. Similarly, node $n_{2}$ can work in the same way to obtain $x_{1}$.

It can be easily seen that the proposed application of soft network coding in wireless cooperative networks performs in a similar way to the amplify-and-forward relay protocol in a normal relay network since the relay node forwards the soft information to other nodes. Thus, the proposed scheme achieves the advantages of both the network coding technique and the amplify-and-forward technique. In particular, when the received signal at the relay node has a lower SNR and could not be decoded correctly, the relay transmission with network coding can still be performed. It can be proved that in this case, the achievable bit rate of the proposed scheme is larger than the conventional scheme in [8]. Another advantage of the proposed scheme is that channel decoding is not necessary at the relay node and the computational complexity at the relay node can be greatly reduced. Similar, the proposed soft network coding can also be applied in the wireless network in [17] to improve the diversity of distributed antenna systems. The details of these applications of soft network coding will be shown in our future work.

\section{CONCLUSION}

Network coding is a promising technique for improving the throughput and robustness in packet networks. Recent research has shown that it can improve the wireless network performance even more significantly. In this paper, we have considered the joint design of network coding and channel coding in wireless networks. In particular, we have introduced the concept of soft network coding, where the LLRs of the network coded bits are generated by combining the soft decisions of the input packets. We have shown that the proposed scheme can achieve almost the same channel capacity as the traditional network coding scheme under moderate to high SNR values. We have also shown that with only 0.5 to $1.0 \mathrm{~dB}$ penalty, the proposed scheme reduces the channel decoding complexity by almost 50\%. Finally, it has been shown that the soft network coding can be easily applied in wireless cooperative networks.

\section{ACKNOWLEDGEMENT}

This work was supported by the Competitive Earmarked
Research Grant (Project Number 414305) established under the University Grant Committee of the Hong Kong Special Administrative Region, China.

\section{REFERENCE}

[1] R. Ahlswede, N. Cai, S.-Y. R. Li, and R. W. Yeung, "Network information flow," IEEE Trans. Inform. Theory, vol. 46, no. 4, pp. 1204-1216, Jul. 2000.

[2] S.-Y. R. Li, R. W. Yeung, and N. Cai, "Linear Network Coding," IEEE Trans. Inform. Theory, vol. 49, no. 2, pp. 371-381, Feb. 2003.

[3] Y. Wu, P. A. Chou, and S.-Y. Kung, "Minimum-energy multicast in mobile ad hoc networks using network coding," IEEE Trans. Commun., vol. 53, no. 11, pp. 1906-1918, Nov. 2005.

[4] J. E. Wieselthier, G. D. Nguyen, and A. Ephremides, "On construction of energy-efficient broadcast and multicast trees in wireless networks," in Proc. IEEE Conf. Comput. Commun., pp. 586-594, Israel, 2000.

[5] S. Deb, M. Effros, T. Ho, D. R. Karger, R. Koetter, D. S. Lun, M. Medard, and N. Ratnakar. "Network coding for wireless applications: A brief tutorial," in Proc. Intern. Workshop on Wireless Ad-hoc and Sensor Networks (IWWAN), 2005.

[6] S. Katti, D. Katabi, W. Hu, H. Rahul, and M. Medard, "The importance of being opportunistic: Practical network coding for wireless environments," Allerton, 2005.

[7] J. Widmer, C. Fragouli, and J.-Y. Le Boudec, "Low-complexity energy-efficient broadcasting in wireless ad-hoc networks using network coding," in Proc. Workshop on Network Coding, Theory, and Applications, Apr. 2005.

[8] C. Hausl and J. Hagenauer, "Iterative network and channel decoding for the two-way relay channel," in Proc. IEEE ICC'06, Istanbul, Turkey, June 2006.

[9] C. Hausl and P. Dupraz, "Joint network-channel coding for the multiple-access relay channels," in Proc. Intern. Workshop on Wireless Ad-hoc and Sensor Networks (IWWAN), New York, USA, June 2006.

[10] S. Zhang, S. Liew, and P. Lam, "Physical layer network coding," in Proc. ACM MobiCom’06, pp. 358-365, LA, USA, 2006.

[11] L. Bahl, J. Cocke, F. Jelinek, and J. Raviv, "Optimal decoding of linear codes for minimizing symbol error rate," IEEE Trans Inform. Theory, pp. 284-287, Mar. 1974

[12] P. Robertson, P. Hoeher, and E. Villebrun, "Optimal and suboptimal maximum a posteriori algorithms suitable for turbo decoding," Europ. Trans. Telecommun., vol. 8, no. 2, pp. 119-125, Mar./Apr. 1997.

[13] J. G. Proakis, Digital Communications, 3rd ed. New York: McGrawHill, 1995.

[14] T. M. Cover and J. A. Thomas, Elements of Information Theory. New York: Wiley, 1991.

[15] S. J. MacMullan and O. M. Collins, "The capacity of orthogonal and bi-orthogonal codes on the Gaussian channel," IEEE Trans. Inform. Theory, vol. 44, no. 3, pp. 1217-1322, May 1998.

[16] Y. Yasuda, K. Kashiki, and Y. Hirata, "High-rate punctured convolutional codes for soft decision Viterbi decoding," IEEE Trans. Commun., vol. 32, no. 3, pp. 315-319, Mar. 1984.

[17] Y. Chen, S. Kishore and J. Li, "Wireless diversity through network coding,” in Proc. IEEE WCNC 2006, pp. 1681-1686, Apr. 2006. 